\title{
Keywords Index
}

3-D dynamometry 414

3-D motion analysis 354

4-Aminopyridine 7

4-aminopyridine 728

Abdominal radiograph 331

Activated protein C 754

Activities of daily living 185

Acute aortic dissection 702

Acute spinal cord compression 403

Acute spinal cord injury 538

Adult spine 766

Aerobic 16

Age 331

Aging 77

Amyloidosis 327

Ankylosing spondylitis 117

Anticholinergics 250

Anxiety 309

Aortic cross-clamping 153

Arrested hydrocephalus 495

ASIA impairment scale 7

Autonomic dysreflexia 229, 581

Axon 728

Backache 63

Baclofen pump 126

Bacterial contamination 100

Bacteriuria 106

Balance 425

Biceps brachii nerve 403

Bioactive glass-ceramic 315

Biochemical markers 211

Biomechanics 649

Bladder auto-augmentation 59

Bladder management 762

Bladder stimuli 229

Bone 622

Bone marrow stromal cells 622

Bone mineral density 26

Bowel function 301, 573, 717

Bowel outcomes 301

Brain abscess 265

Brown-Séquard syndrome 705

Calculi 346

Carboxyterminal propeptide of type I collagen 211

Carboxyterminal telopeptide of type I collagen 211

Cardio-respiratory 16

Carers 559

Catheterization 45, 97

Cauda equina 615
Causes of death 604

Central cord syndrome 120, 532

Central pain 611,658

Cerebellar haemorrhage 504

Cerebrospinal fluid 504

Cerebrospinal fluid shunts 495

Charcot joint 112

Children 622

Chronic constipation 301

Chronic pain syndrome 63

Chronic spinal cord injury 733

Classification 675

Clinical 449

Colon 615

Colonic transit 301

Colonic wash-out 255

Compression fracture 639

Computer modeling 473

Congenital kyphosis 382

Constipation 255, 331, 615

Conus medullaris infarction and medullary vein fistula 711

Corticospinal physiology 292

Creatinine clearance 197

Credé maneuver 552

Cruciate paralysis 120

Cycle ergometer 214

Cycling 680

Deltoid triceps transfer 435

Demography 164

Depression 309, 559

Dexamethasone 273

Diagnosis 203

Dipstick test 106

Disability evaluation 234

Disc degeneration 708

Divorce 2

Dorsal root response 287

Drainage 504

DuoDERM $^{\circledR} 100$

Dyaesthesia 611, 658

Education 173

Electric stimulation 724

Electrode position 473

Electrophysiological recordings 389

Employment 51, 173

En bloc resection 146

Enema 255

Energy cost of locomotion 37

Enterocystoplasty 490

Eosinophilic granuloma 766
Epidemiology 164, 346, 420, 597, 697

Epididymo-orchitis 769

Erectile dysfunction 363

ESWL 325

Etiology 164

Evoked potentials 754

Exercise 680

Experimental 449

Faecal incontinence 255, 573, 717

Failed back 63

Fatigue 214

Fecal incontinence 724

FES 244, 680

Fibrosis 442

Functional 120

Functional capacity impairment 414

Functional electrical stimulation 630

Functional independence 435

Functional neuromuscular stimulation 37

Functional outcome 185

Funiculitis 769

Gentamicin 197

Grey matter 403

H-reflex 287

Handfunction 244

Heat exposure 224

Hematoma 192

Hemilaminectomy 92

Heterotopic ossification 33, 319

Histology 45

Hong Kong Chinese 687

Horner's syndrome 705

Hospitalisation 371

Hydronephrosis 661

Hyperbaric oxygen therapy 538

Hypogastric artery 711

Hypotension 741

Immunoglobulin A 378

Immunohistochemistry 546

Incidence 420

Incomplete spinal cord injury 292

Incontinence 59, 262, 490

Infection 126

Intra-operative monitoring 133

Intracranial pressure 495

Intramedullary tumour 504 
Intrathecal 728

Intrathecal gas 708

Irradiation 319

Ischial pressure sore 571

Isometric torque 414

Kidney-calculi 325

Kidney-calculus 661

Kurtzke Functional Systems Scales 409

Laminae 442

Laminectomy 442

Laminotomy 442

Langerhans' cell histiocytosis 766

Laparoscopy 59

Laxatives 331

LBP 414

Lesion size 754

LoFric $^{\circledR} 45$

Long-term follow-up 563

Loose body 112

Low back pain 63

Lumbar disk 63

Lumbar spinal cord 394, 473

Lumbar spine 315

Marriage 2

Mean response time 340

Measurement 675

Measurements 509

Megacolon 331

Methylprednisolone 71, 273, 733

Microdialysis 153

Midodrine hydrochloride 109

Milk of calcium 325

MK 801733

Mood 309

Morbidity 563

Morphometry 532

Mortality 563

Motility 615

Motion analysis 683

Motor disturbance 754

Motor nerve conduction study 203

Motor neurons 532

MRI 56, 117

Multiple sclerosis 409

Muscle characteristics 214

Muscle metabolism 630

Muscle strength ratios 748

Muscular exercise 16

Myelomeningocele 495

Myelopathy 382

Near-infrared spectroscopy 158

Nephrolithiasis 346

Nephrotoxicity 197

Neural plasticity 728

Neurogenic bladder 250, 262, 490
Neurologic complications 133

Neurological assessment 292

Neuropathic bladder 97, 378, 546, 762, 769

Neuropathic bowel 301

Neuropathic joint 112

Neuroprosthetics 724

Neurostimulation 262

Neurotrophic joint 112

Nimodipine 71

Nocardia 265

Odontoid fracture 117

OPLL 211

Orthostatic 741

Orthostatic hypotension 109

Osmolality 45

Ossification of the posterior longitudinal ligament 140

Osteoblast 622

Osteocalcin 211

Osteoconduction 315

Osteonecrosis 319

Osteoporosis 639

Outcome 120, 567

Outcome assessment 84

Oxygen uptake kinetics 340

Pain 611, 658

Painless 702

Paraplegia 37, 100, 112, 203, 214, 571, 622, 630, 649, 702, 741, 748

Paraplegic 16

Paraplegic classification 234

Paraplegics 224

Partner relationships 2

Peripheral nerve evoked potentials 403

Peripheral quantitative computed tomography 26

Personal and marital adjustment 687

Personality 555

Persons with SCI 687

Physical activity 26

Physical therapy 741

Pig 153

Placement of electrode 394

Plain radiography 315

Pointing movements 354

Portapres 158

Post discharge 498

Post-traumatic syringomyelia 567

Postero-lateral fusion 315

Postural control 748

Posture 425

Pott's paraplegia 669

Prediction 185

Prehension 354

Pressure ulcer 100
Primary vertebral tumor 146

Prognosis 203

Psychological morbidity 559

Psychological stress 555

Psychological wellbeing 559

Psychometric quality 509

PTHrP 546

Public health 597

Pyogenic vertebral osteomyelitis 639

Pyonephrosis 661

Pyridinoline 211

Quadriplegia 445, 630

Quality of health care 84

Quality of life 363, 498, 509

Radiotherapy 33

Rats 733

Reaching 354

Readmission 371

Reciprocating gait orthosis 37

Reconstructive hand surgery 541

Recovery 120, 680

Recreation 173

Recruitment order 473

Rectum 615

Recurrence 571

Regeneration 449

Registries 597

Rehabilitation 51, 84, 117, 185, 762

Reliability 675

Removal friction 45

Renal failure 604

Research 597

Residual urine volume 409

Respiratory mechanics 445

Respiratory tract infection 604

Review 509

RGO 555

Rhizotomy 262

Rolling 683

Rotation flap 571

Rotator cuff disorders 748

SCI 77, 120, 273, 555

Sciatica 63

Self catheterisation 409

Self-care 97

Septicaemia 604

Shoulder 649

Sitting position 425

Skin blood flow 224

Skin temperature 224

Slow ascending myelopathy 327

Somatosensory evoked potentials 133

Spasticity 26, 287, 389, 524

Spina bifida 255

Spinal arteriography 711 
Spinal cord 97, 717

Spinal cord evoked potentials 403

Spinal cord injuries 100, 597

Spinal cord injury 2, 7, 26, 33, 51, $71,84,106,109,120,140,164$, $173,185,197,234,244,255,301$, $309,319,325,331,340,346,354$, $363,371,378,389,420,435,449$, 490, 498, 509, 552, 559, 563, 567, $573,604,611,615,658,661,675$, $680,683,697,705,717,724,728$, 741, 754, 762, 769

Spinal cord ischaemia 153

Spinal cord stimulation 394, 473, 524

Spinal dural arteriovenous fistulas 711

Spinal injury 229

Spinal instability 92

Spinal ischemia 702

Spinal lesions 92

Spinal shock 229

Spinal stenosis 140

Spinal subdural hematoma 192

Spinal surgery 133

Spinal tumour 639

Spine 56

Spirometry 445

Spontaneous 192
Sport 16

Sports 173

Sports activity 309

Spouses 687

Squamous carcinoma 327

Squamous metaplasia 546

Subdural abscess 56

Suicide 604

Surgery 319

Syringomyelia 265

Tetraparesis 120

Tetraplegia 100, 158, 164, 197, 244, $327,354,425,435,541,649,683$, 741,762

The Netherlands 420

Thecal cyst 708

Total en-bloc spondylectomy 382

Total spondylectomy 146

Tracheal stenosis 445

Trauma 120, 538

Traumatic 420

Treatment 389

Trunk stability 748

Tuberculosis 56, 669

Turkey 697

Tympanic membrane temperature 224
Upper extremity 649

Upper lumbar spinal cord segment 524

Urethra 45

Urinary tract infection 661

Urine culture 106

Urine microscopy 106

Urodynamics 250, 490

Urologic complications 552

Urological management 409, 541

Urothelial hyperplasia 546

Urothelium 378

Urothelium-differentiation 546

Vastus lateralis 630

Ventral root response 287

Vertebra plana 766

VIAGRA $^{\mathbb{B}} 363$

$\dot{\mathrm{V}} \mathrm{O}_{2} 37$

Vocational 51

Voluntary function 680

Walking index 234

Wheelchair 37, 425

White matter 403

Xanthogranulomatous inflammation 769 